\title{
Automatic segmentation of kidney and kidney tumor based on 3D convolutional neural networks
}

\author{
Pengchong Qiao, Bin Zhao, and Zhiyang Liu \\ Nankai University, Tianjin 300071, China
}

\begin{abstract}
Kidney cancer is a huge threat to humans, and the surgery is the most common treatment. For clinicians, knowing the morphology of the kidney and kidney tumor in advance may be helpful for surgery. Automatic segmentation of kidney and kidney tumor is a promising approach for these efforts. In this paper, we proposed a based 3D convolutional neural network to segment kidney and kidney tumor using the data from the 2019 Kidney Tumor Segmentation Challenge.
\end{abstract}

Keywords: deep learning - medical imaging - semantic segmentation.

\section{Introduction}

There are more than 400,000 new cases of kidney cancer each year [1], and surgery is its most common treatment [2]. For clinicians, knowing the morphology of the kidney and kidney tumor in advance may be helpful for surgery. However, manual segmentation of kidney and kidney tumor from CT-Volumes is expensive in terms of time and human expertise. Automatic segmentation is the much desired solution to this problem.

Following the success on challenging tasks in the field of computer vision [3, 4]. Convolutional Neural Networks (CNNs) have been applied successfully on variety of biomedical segmentation problems. Most developed approaches relied on the adaptation of 2D CNNs for processing 3D volumes [5, 6]. However, these methods dropped context information when they are trained slice by slice. Therefore, the approaches based 3D CNNs have been proposed for solving the problem [7-9]. In this paper, we proposed a method based on 3D CNNs for segmentation of kidney and kidney tumor using the data from the 2019 Kidney Tumor Segmentation Challenge (KiTS19).

\section{Methods}

The KiTS19 challenge released 209 cases for model training, which is the format of CT-Volumes including subjects and corresponding manual delineation of the actual kidneys and kidney tumors. The overview of our proposed method is shown in the Figure 1. Our proposed method consists of two auxiliary networks and a segmentation network. The auxiliary networks are used to locate the kidneys in advance. As Fig. 1 shows, the one based resnet34 is for obtaining the range of axis $\mathrm{z}$ of the case, and the one based unet-2d is used to locate kidney in the slice of axis $\mathrm{x}$ and axis $\mathrm{y}$. We acquire $3 \mathrm{D}$ patches with the size of $(128,128,80)$ by adjusting the sliding window from the located kidney. Hereafter, these 3D patches are input the segmentation network for training the parameters. The proposed segmentation network is shown in Fig.2. To acquire more semantic features in the lower layers, more transpose convolution layers are added in segmentation network. Besides, multiple scale residual blocks (MRB) are used to obtain multiple scale features, which present plentiful context information for increasing the accuracy of segmentation. The MRB is shown in Fig. 3. As the Fig.3 shows, the MRB uses cascade of 3x3 kernel convolution layer to obtain a bigger receptive field, then concatenates all the features with a residual shortcut. There is a $1 \times 1$ convolution layer used for dimensionality reduction of the features in each MRB. 


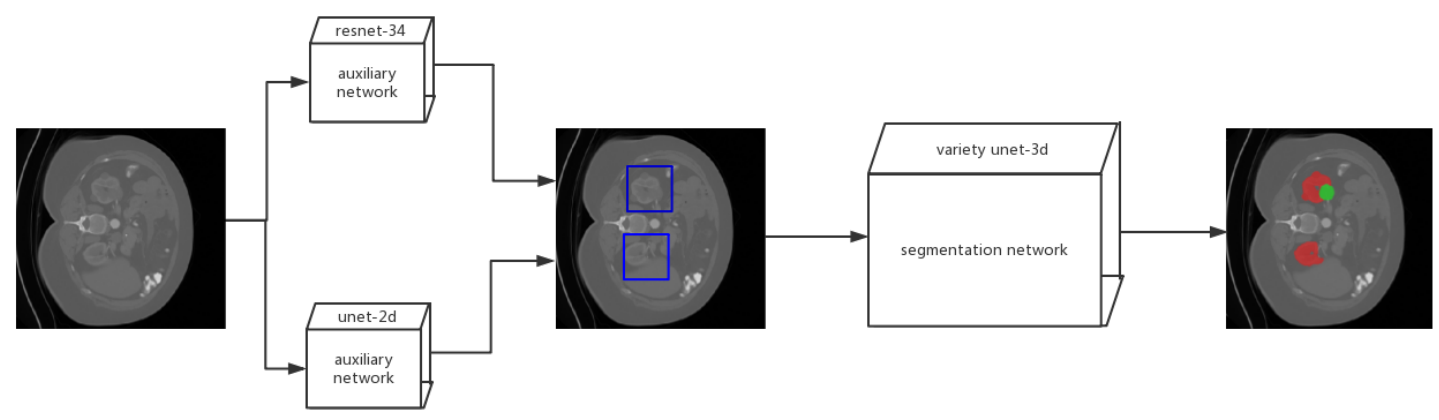

Fig.1. Overview of the segmentation process.

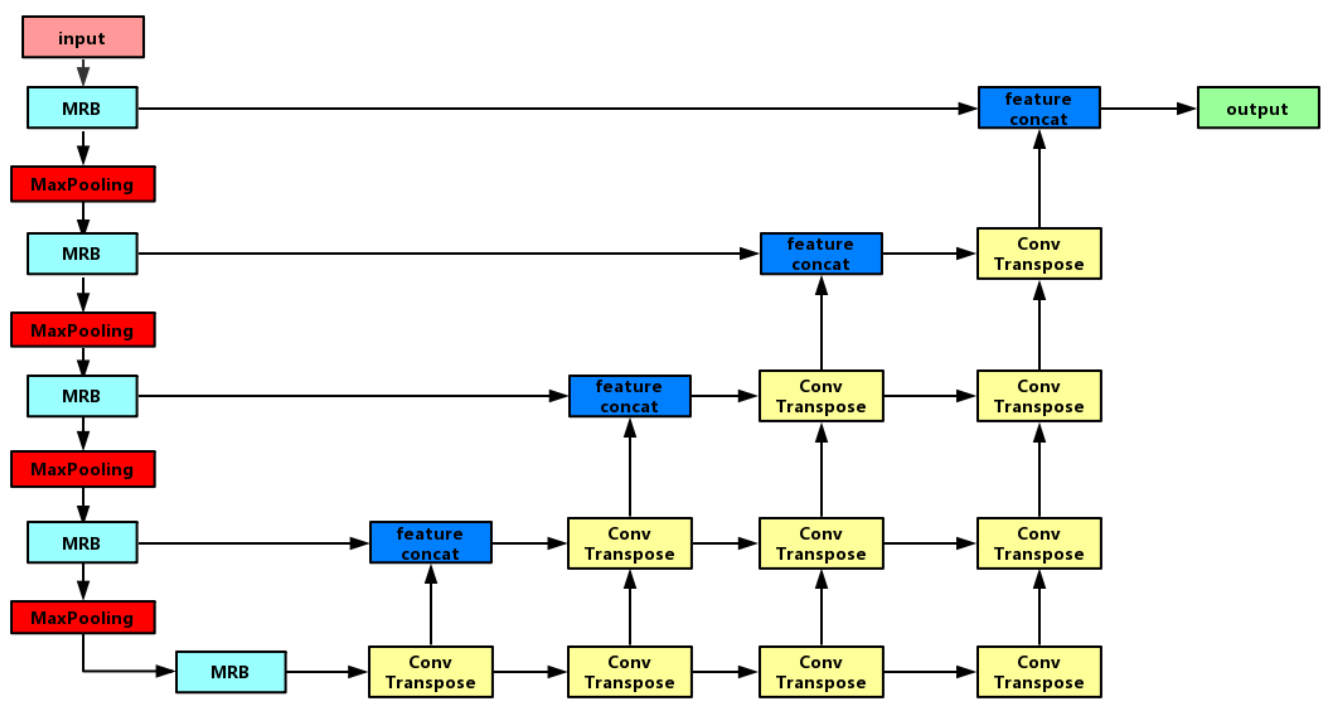

Fig. 2. Segmentation network 


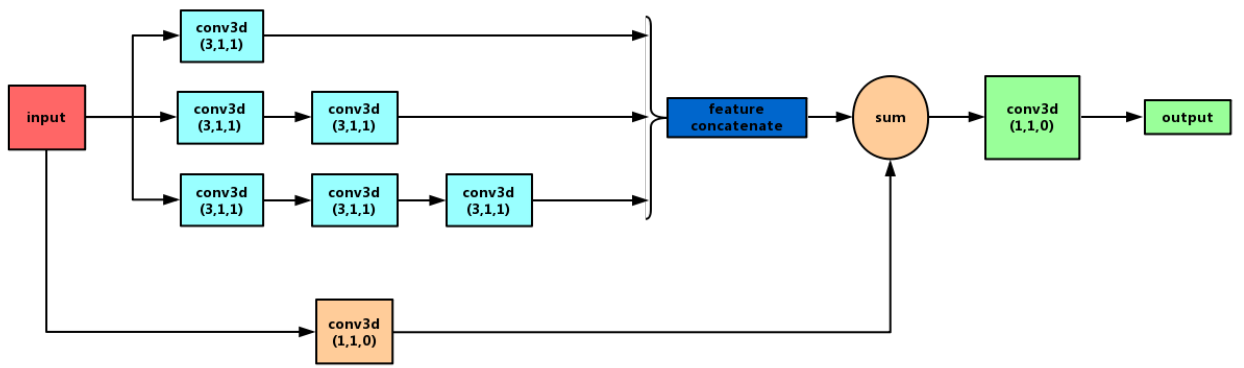

Fig. 3. Multiple scale Residual Block

\section{Implementation details}

By setting the threshold of 350, the values of pixels below the threshold will be set to zero. Meanwhile, We use Trilinear interpolation to make all the spacing normalized to $(1,1,1)$. In particular, each input image is randomly rotated by a degree ranging from -30 ॰ to 30 ॰ degree, flipped randomly on the fly, so as to augment the dataset and reduce memory footprint. We use SGD as optimizer with initial learning rate of 0.01 and decay by 0.1 if no progress is made in three epoch. Early-stopping technique is adopted after 30 epochs or the learning rate is lower than 1e-6 with no progress on the validation loss. We use the summation of softdiceloss and Binary Crossentropy Loss as our loss function.

\section{References}

[1] “Kidney Cancer Statistics.” World Cancer Research Fund, 12 Sept. 2018. Available: www.wcrf.org/dietandcancer/cancer-trends/kidney-cancer-statistics.

[2] "Cancer Diagnosis and Treatment Statistics." Stages | Mesothelioma | Cancer Re- search UK, 26 Oct. 2017. Available: www.cancerresearchuk.org/health-professional/cancerstatistics/diagnosis-and-treatment.

[3] A. Krizhevsky, I. Sutskever, and G. E. Hinton, "ImageNet Classification with Deep Convolutional Neural Networks," Neural Information Processing Systems, vol. 25, 01/01 2012.

[4] S. Ioffe and C. Szegedy, "Batch normalization: accelerating deep network training by reducing internal covariate shift," presented at the Proceedings of the 32nd International Conference on International Conference on Machine Learning - Volume 37, Lille, France, 2015.

[5] Z. Liu, C. Cao, S. Ding, Z. Liu, T. Han, and S. Liu, "Towards Clinical Diagnosis: Automated Stroke Lesion Segmentation on Multi-Spectral MR Image Using Convolutional Neural Network," IEEE Access, vol. 6, pp. 57006-57016, 2018.

[6] L. Chen, P. Bentley, and D. Rueckert, "Fully automatic acute ischemic lesion segmentation in DWI using convolutional neural networks," Neuroimage Clin, vol. 15, pp. 633-643, 2017.

[7] K. Kamnitsas, L. Chen, C. Ledig, D. Rueckert, and B. Glocker, "Multi-scale 3D convolutional neural networks for lesion segmentation in brain MRI," Ischemic Stroke Lesion Segmentation, pp. 13-16, 2015.

[8] K. Kamnitsas et al., "Efficient multi-scale 3D CNN with fully connected CRF for accurate brain lesion segmentation," Med Image Anal, vol. 36, pp. 61-78, Feb 2017. 
[9] R. Zhang et al., "Automatic Segmentation of Acute Ischemic Stroke From DWI Using 3-D Fully Convolutional DenseNets," IEEE Trans Med Imaging, vol. 37, no. 9, pp. 2149-2160, Sep 2018. 